\title{
Robust Spectrum Allocation for Cognitive Radio Networks
}

\author{
Germán Capdehourat, Federico Larroca and Pablo Belzarena \\ Instituto de Ingeniería Eléctrica, Facultad de Ingeniería, Universidad de la República, Uruguay \\ \{gcapde@fing.edu.uy, flarroca@ fing.edu.uy, belza@fing.edu.uy\}
}

\begin{abstract}
In recent years we have seen the success of wireless communications in unlicensed frequency bands, mainly driven by the 802.11 standard. The clear trend in the growth of traffic demand suggest that spectrum scarcity will be a serious threat to reach the capacity needs in the near future. Looking for more available spectrum, regulators have already begun to study secondary assignments in licensed bands, based on the recent cognitive network paradigm. In this context, we focus our work in the analysis of optimum spectrum allocation mechanisms. We introduce a stochastic model to formulate the problem, considering primary users' activity and a periodically scheduled assignment scheme. To solve the problem we propose a novel robust solution, which we argue is superior to an expectation based approach, comparing both alternatives through extensive simulations.
\end{abstract}

\section{INTRODUCTION}

Over the last years we have witnessed the highest growth in wireless networks traffic [1] and forecasts indicate that this growth will continue [2]. Moreover, the user density is also increasing, resulting in scenarios where the technology is reaching its limits. Examples include the one-to-one educational model (e.g. Plan Ceibal [3]) with thirty or more mobile devices in the same room, or crowded places as large conferences, shopping centers or even sport events (e.g. the last Super Bowl [4]). While standards are still evolving, achieving increasingly higher spectral efficiency, we may soon be faced with spectrum scarcity issues to properly cope with traffic demands. Regulators have taken note about this fact and some proposals already exist to extend the available spectrum [5].

Leaving aside traditional spectrum allocation, during the last years a new type of spectrum assignment has emerged: the so-called cognitive radio paradigm [6]. The main idea is to have two types of users; licensed or primary users (PUs from now on), which have the preferential right to use the band; and unlicensed or secondary users (SUs from now on), which can use the band only in the absence of the PUs. This type of spectrum allocation contributes to a more efficient use compared to traditional static assignments, as testified by some recent FCC rulings [7].

Although adoption is not yet massive, much industrial and academic efforts have been dedicated to this kind of technology. For instance, the IEEE 802.22 standard [8] was approved in 2011, which defines a Wireless Regional Area Network (WRAN) based on cognitive radio. Another industrial effort is the 802.11af amendment to enable the operation of
WiFi in TV bands, which has been recently published [9]. It is then expected that, as it has been so far for 802.11, networks deployed using these standards will mostly operate on infrastructure mode, so we will focus on such networks.

This paper bears on the dynamic spectrum assignment in this kind of networks. That is to say, we will study possible methods to decide which frequency bands may be used by the network devices at any given time. It is worth to highlight that such an assignment means that the bands are available for the devices, and are not necessarily used. With this in mind, the natural question that arises is to what purpose this assignment should be performed [10]. In our particular context, examples include minimizing the number of licensed bands assigned [11] or maximizing the user's utility (as a function of the mean rate) [12] without exceeding a maximum interference threshold to other networks. However, in the context of a cognitive WRAN or WLAN, we argue that the most natural objective would be to provide a lower bound to the resulting throughput. A typical use case for these technologies is internet access, usually following a wired connection, which is limited to a certain fixed bandwidth. The purpose of the spectrum allocation is to ensure this bandwidth can always be fully exploited, no matter what the channel conditions are or which the PU's activity is. Therefore it should be a constraint of the problem.

The other challenge that these systems pose is the timescale at which the assignment should be performed. One possibility is to re-assign (and thus re-optimize) every time a band is used or abandoned by PUs, or if significant changes in channel conditions occur. Although this event-driven solution will lead the system to operate with the optimal allocation all the time, it will typically result in a higher signaling overhead. In this sense, we will assume, as many researchers, a periodic optimization every $T$ time units, which leads us to a better performance compromise.

However, $T$ may include variations in PUs' activity. This fact implies that a licensed band assigned when the period starts might have to be abandoned, resulting in an effective capacity that is less than expected. In Fig. 1 we present an example to clarify this situation. In it we have four licensed bands, with two of them available at the first spectrum assignment at time 0 . During the interval between allocations, a PU starts using band 4, so it is no longer available. The problem occurs again in the second assignment, where bands 


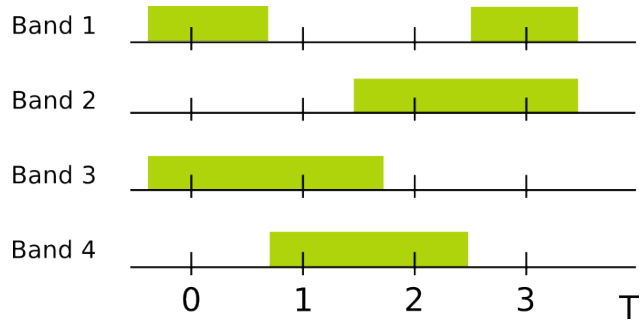

Fig. 1. Spectrum occupancy example with four licensed bands.

1 and 2 are available and the assignment is thus performed, but a PU occupies band 2 during the interval.

To address this issue, the most commonly used approach is to model the availability of licensed bands as random, and optimize the expected value of indicators such as interference or throughput, as discussed before. Although this means that in the long run the objective will be accomplished (e.g. the throughput will be maximized), in the shorter time-scales the resulting performance may be far from optimal. In contrast with previous works, we will present a frequency assignment scheme that provides the required throughput, which will hold with very high probability during the whole operating time. Naturally, such guarantee will require a certain degree of overprovisioning, but our simulations indicate that this is usually below $15 \%$ of that required by an oracle that knows beforehand the PUs activities. Moreover, the results show that simply considering an expected value approach leads us to a solution where the throughput requirement is not fulfilled more than $30 \%$ of the time.

\section{Problem Formulation and Proposed Solutions}

In this paper we study the spectrum allocation problem in a mixed licensed and unlicensed scenario. In the proposed scheme, devices operate always as unlicensed devices but in two types of frequency bands, licensed ones, where they are only allowed to operate when there is no PUs, and unlicensed ones, where they can operate all the time. This offers greater flexibility to meet the requirements, given the scarcity of unlicensed spectrum. Furthermore, by having both type of bands, we simplify some protocol design complexity compared to solutions which only use licensed bands, as we can perform control communications through unlicensed bands, which are available all the time. To accomplish this goal we will impose that any possible assignment should include at least one unlicensed band. This way we ensure the connectivity between nodes which makes possible the proper coordination for the use of the allocated frequency bands.

As in other previous works (e.g. [13]) we will assume that each node has a dedicated interface to enable cognitive sensing capabilities. By this mean, each node is able to keep a record for the PUs' activity on each licensed band. Besides, this interface is used to collect air measurements data, which are used to estimate the available capacity on each band, either licensed or unlicensed. This effective capacity depends on several factors such as channel conditions and other SUs' activity (devices from other networks that are not under our control), but it can be estimated passively through measurements [14] [15]. We consider a solution where the assignment is performed every $T$ time units and we will further assume that $T$ is realatively small, so that an accurate estimation of each band's available capacity may be obtained using information from the previous interval. In this work we suppose that such estimation is exact, so as to concentrate only in the PUs' dynamics.

We will also assume that devices can fully exploit the available spectrum (even disjoint available bands), using a PHY layer such as OFDM. We also assume there is a MAC layer mechanism in order to share the spectrum between nodes (e.g. 802.11 MAC layer). We will focus on a singledomain spectrum assignment, that is to say, a single cell of an infrastructure mode network. For example, a use case scenario would be an access point covering several clients or a point to point link between two nodes.

Let $u=1, \ldots, U$, be the set of unlicensed frequency bands (i.e. no PUs, as in ISM bands). Let $b=1, \ldots, B_{t}$, be the set of licensed frequency bands (which are assigned to a PU) available at time $t$ (i.e. PUs are not present). We will note as $c_{b}(t)$ the effective capacity available on licensed frequency band $b$ and $c_{u}(t)$ the effective capacity available on unlicensed frequency band $u$. We define as spectrum assignment variables $\alpha_{b}(t)$ and $\alpha_{u}(t)$, which belong to [0,1], assuming partial band assignment is possible (e.g. via OFDMA or TDMA).

Now, we can define the total capacity assigned for the interval starting at $T$ as:

$$
C\left(\alpha_{T}\right)=\sum_{b=1}^{B} \alpha_{b}(T) c_{b}(T) h_{b}(T)+\sum_{u=1}^{U} \alpha_{u}(T) c_{u}(T)
$$

where $h_{b}(T)$ is a real number in $[0,1]$, according to how much time each licensed band was actually available during the interval. We will model $h_{b}$ as a random variable, whose distribution will be learnt from the previously observed dynamics. As we stated previously the objective is to provide a lower bound to the resulting throughput, so we will set this bound as a problem constraint, and we shall note it as $d$. This lower bound $d$ is actually the minimum total capacity our system should have considering all nodes. We further define a cost function:

$$
\mathcal{C}\left(\alpha_{t}\right)=\mathcal{C}_{\text {lic }}\left(\alpha_{1}(t), \ldots, \alpha_{B}(t)\right)+\mathcal{C}_{\text {unlic }}\left(\alpha_{1}(t), \ldots, \alpha_{U}(t)\right)
$$

The cost functions $\mathcal{C}_{\text {lic }}()$ and $\mathcal{C}_{\text {unlic }}()$ allow us to give different weights for each band, depending on the desired spectrum allocation goal. For example, it is possible to have different costs depending if the band corresponds to a higher or lower frequency, which may imply different transmission power requirements.

After all the stated assumptions, definitions and goals, we can now define an optimization problem which will lead us to the assignment algorithm. This problem should be solved periodically each time $T$, so we will omit the time index from now on for a matter of clarity. That is to say, each time $T$ we 
should strive at solving the following problem:

$$
\begin{array}{cl}
\underset{\alpha}{\operatorname{minimize}} & \mathcal{C}_{\text {lic }}\left(\alpha_{1}, \ldots, \alpha_{B}\right)+\mathcal{C}_{\text {unlic }}\left(\alpha_{1}, \ldots, \alpha_{U}\right), \\
\text { subject to } & \sum_{b=1}^{B} \alpha_{b} c_{b} h_{b}+\sum_{u=1}^{U} \alpha_{u} c_{u} \geq d, \\
& \sum_{u=1}^{U} \alpha_{u} \geq 1, \\
& \alpha_{b} \in[0,1], b=1, \ldots, B, \\
& \alpha_{u} \in[0,1], u=1, \ldots, U .
\end{array}
$$

The problem above is actually not well defined, as $h_{b}$ is a random variable. To take into account this fact, the first and, as discussed in the introduction, most common approach, is to use the expected capacity, which is simply:

$$
\bar{C}(\alpha)=\sum_{b=1}^{B} \alpha_{b} c_{b} \mathrm{E}\left\{h_{b}\right\}+\sum_{u=1}^{U} \alpha_{u} c_{u} \geq d,
$$

where $\mathrm{E}\left\{h_{b}\right\}$ can be estimated from the previous records of the PU's activity. Thus, the problem above is convex (assuming the defined cost functions are convex) and can be solved with standard optimization tools.

The alternative is to change the expected effective capacity constraint for a probabilistic one:

$$
\operatorname{Prob}\left(\sum_{b=1}^{B} \alpha_{b} c_{b} h_{b}+\sum_{u=1}^{U} \alpha_{u} c_{u} \geq d\right) \geq 1-\epsilon,
$$

where $\epsilon$ is a fixed value (close to 0 ), which leads us to a convex chance constrained optimization problem (assuming $h_{b}$ has a log-concave distribution). This approach, with the advantage of being robust, is more difficult to solve in the general case. In our particular case it is possible to use the distributionally robust deterministic equivalent proposed in [16], which is defined by $h_{b}$ 's means and variances, values that can be estimated from the previous records of the PU's activity. This way, we have again a convex optimization problem, but now with a deterministic equivalent constraint:

$$
\sum_{b=1}^{B} \alpha_{b} c_{b} \mathrm{E}\left\{h_{b}\right\}+\sum_{u=1}^{U} \alpha_{u} c_{u}-\kappa_{\epsilon} \sum_{b=1}^{B} \alpha_{b} c_{b} \sqrt{\operatorname{Var}\left\{h_{b}\right\}} \geq d
$$

where $\kappa_{\epsilon}=\sqrt{1-\epsilon / \epsilon}$. By this equivalence the problem can also be solved by standard optimization tools.

\section{Simulation EXPERIMENTS}

In order to model and simulate the licensed band's scenario under study, we considered a two-state On-Off discrete time markov chain (DTMC) spectrum occupancy model (see Fig. 2), which has been proved to be suitable [17]. The parameters involved in the model are the transition probabilities $\mathrm{p}_{\text {on }}$ and $\mathrm{p}_{\text {off }}$, which will determine the average busy and non-busy time, $\pi_{\text {on }}=\mathrm{p}_{\text {on }} /\left(\mathrm{p}_{\text {on }}+\mathrm{p}_{\text {off }}\right)$ and $\pi_{\text {off }}=\mathrm{p}_{\text {off }} /\left(\mathrm{p}_{\text {on }}+\mathrm{p}_{\text {off }}\right)$, respectively. While it is not necessary for the implementation of the algorithms, as a measurement-based estimation is sufficient, it

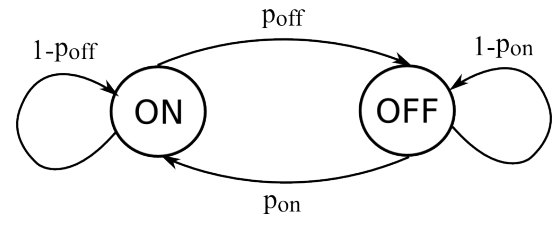

Fig. 2. Two-state On-Off DTMC spectrum occupancy model.

is possible with this model to obtain closed-form expressions of $E\left\{h_{b}\right\}$ and $\operatorname{Var}\left\{h_{b}\right\}$ from the model parameters.

We considered a total of 50 frequency bands for all the simulations, performed for different scenarios. The effective capacities for each band were all drawn once from a uniform distribution between 5 and $25 \mathrm{Mbps}$, and remained the same for all the simulations. All bands were considered of equal spectrum bandwidth, each with a generic value BW. Each simulation was performed for a total time period of $1000 \mathrm{~T}$, where $\mathrm{T}$ (also a generic value) is the time interval between spectrum allocations. Finally, we used the DTMC spectrum occupancy model to simulate the PUs' activity, completing a total of 20 transitions during each interval, a fixed value used for all the experiments. The initial occupancy for each licensed band was drawn in all cases from the corresponding stationary distribution $\pi_{\text {on }}$, in order to start each simulation already at steady state. In order to solve the optimization involved in each method we used CVX [18], working with MOSEK [19] as solver.

First, we set 15 unlicensed bands and 35 licensed, and we analyze the algorithm allocation for different values of $\mathrm{p}_{\text {on }}$ and $\pi_{\text {on }}$. Then, we varied the proportion of bands of each type, and we study the algorithm allocation for different values of $d$ (now with fixed $\mathrm{p}_{\mathrm{on}}$ and $\pi_{\mathrm{on}}$ ). As reference results we considered the solution to the proposed problem when the realization of $h_{b}$ is known in advance. We shall call this method the fortune teller (FT). We also include as reference another simple approach to solve the problem, which we shall note as CONS (for conservative), and consists of assigning only unlicensed bands to meet the requirements. It is clear that this assignment is the safer one concerning the PUs, but it has the disadvantage of missing out on using all the available licensed bands. Furthermore, it cannot solve the problem when the unlicensed spectrum is not enough to reach the throughput lower bound. To reference the proposed algorithms, we shall call EXP the one that uses the constraint with the expected effective capacity. On the other hand, we shall call ROB- $\epsilon$ the one that takes the robust deterministic equivalent constraint, where $\epsilon$ is 0.1 or 0.2 .

\section{A. Example: minimize the number of bands assigned}

For the experiments we set as goal to minimize the total assigned spectrum, which might be a suitable objective for SUs, to have a friendly coexistence of multiple devices from different networks, sharing all the available spectrum. This leads us to use the following cost functions: $\mathcal{C}_{\text {lic }}\left(\alpha_{1}, \ldots, \alpha_{B}\right)=$ $\sum_{b=1}^{B} \alpha_{b}$ and $\mathcal{C}_{\text {unlic }}\left(\alpha_{1}, \ldots, \alpha_{U}\right)=\sum_{u=1}^{U} \alpha_{u}$. Anyway, the 

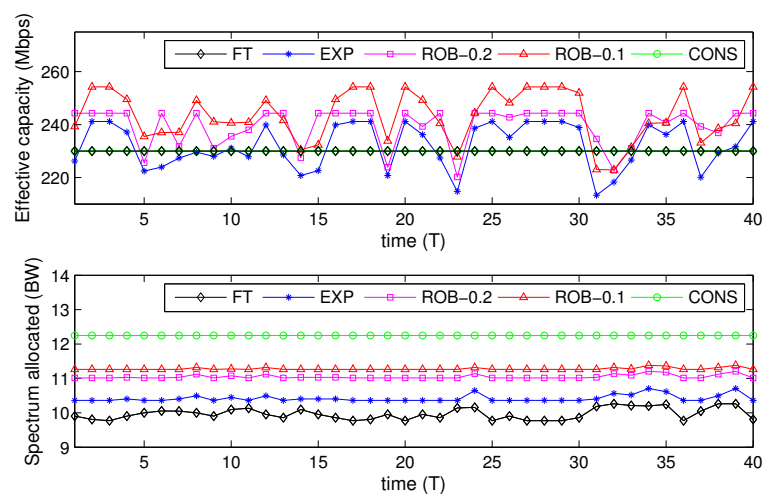

Fig. 3. Experiment example with $\mathrm{d}=230 \mathrm{Mbps}, \mathrm{p}_{\mathrm{on}}=0.01$ and $\pi_{\text {on }}=0.1$.

framework presented is more general and other issues could be considered that would lead to different cost functions.

In Fig. 3 we show an example simulation with parameters $\mathrm{p}_{\text {on }}=0.01$ and $\pi_{\text {on }}=0.1$, the same for all licensed bands. The throughput lower bound $d$ in this case is $230 \mathrm{Mbps}$, somewhat below the total unlicensed bands' capacity which is $248 \mathrm{Mbps}$. Notice that CONS and FT are superimposed in the capacity plot, as they both reach the equality in the constraint. In the experiments we analyze the average effective capacity and spectrum assigned during the simulations lasting $1000 \mathrm{~T}$ (in this example we only show $40 \mathrm{~T}$ ). We also study the short term success of the proposed methods, which is the percentage of intervals where the effective capacity is above the defined lower bound. We will see that although the expected value approach meets the requirements in average, and is the most efficient regarding spectrum usage, robust approaches perform much better at short scales, with a reasonable cost in terms of spectrum bandwidth allocated. In all the simulated situations, only using unlicensed spectrum is enough to meet the requirements, which allows to get a solution with CONS.

We first analyze the results for different values of $\mathrm{p}_{\text {on }}$ (see Fig. 4). As we can see all the methods meet the throughput lower bound in average, something we ensured by placing it as a constraint in the problem formulation. Looking at the spectrum assignment, the stochastic approaches clearly outperform CONS, with better spectral efficiency and closer to the FT optimum solution as soon as $\mathrm{p}_{\text {on }}$ goes to 0 . It is clear that for lower values of $p_{\text {on }}$ is when these methods make better sense, as it indicates higher possibilities of making profit from licensed bands. While robust approaches allocate more spectrum than the expected value solution, in exchange they get much better performance at short scale. The average success rate is $95.4 \%$ and $91.5 \%$, for ROB-0.1 and ROB0.2 respectively, while EXP only gets $72.8 \%$. The extra spectrum assigned in average by robust approaches implies some average capacity overallocation with respect to the stated throughput lower bound. However, this mild conservatism, allocating not much more spectrum than FT, is what enables a higher probability to meet the throughput lower bound also
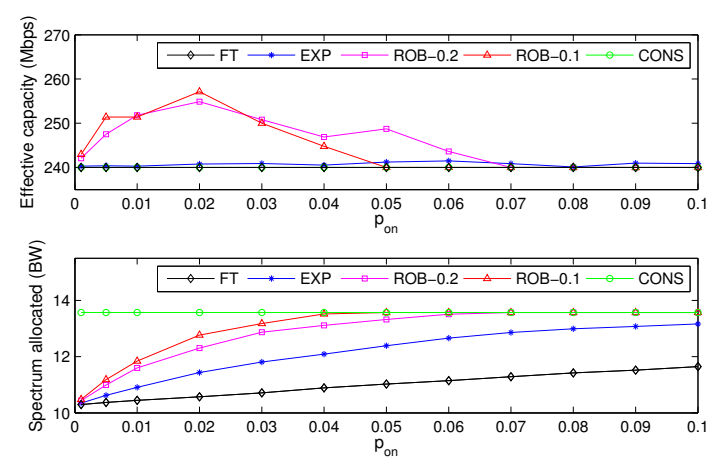

Fig. 4. Effective capacity and spectrum allocated as a function of $\mathrm{p}_{\mathrm{on}}$ when $\pi_{\mathrm{on}}=0.1$ and $d=240 \mathrm{Mbps}$ (total unlicensed capacity $=248 \mathrm{Mbps}$ ).

at the short scale.

Now, we analyze the performance for different busy times (see Fig. 5). We have again a clear advantage of the stochastic methods against CONS, with less spectrum allocated to meet the same requirements. Furthermore, the advantage is higher for lower values of $\pi_{\mathrm{on}}$, which are the most interesting situations to benefit from licensed spectrum. Robust approaches present again some average capacity overallocation, which is higher for lower values of $\pi_{\text {on }}$. In return, their short term effectiveness stands out again, with an average success rate of $86.4 \%$ and $82.6 \%$, for ROB- 0.1 and ROB- 0.2 respectively, against $61.6 \%$ for EXP. This implies that, although the EXP solution meets the requirements in average, almost $40 \%$ of the time the effective capacity assigned is below the stated throughput lower bound. Except for particular cases, where an expectation based solution might be sufficient, we argue instead that a robust approach will be more suitable in practice, with much higher short term performance at a reasonable cost in terms of spectrum.

Lastly, we set as fixed values $\mathrm{p}_{\mathrm{on}}=0.01$ and $\pi_{\text {on }}=0.1$, and we vary the number of unlicensed bands (from 10 to 30), keeping the same total number of bands (50) for all cases. This way, the total unlicensed bands' capacity changes and we
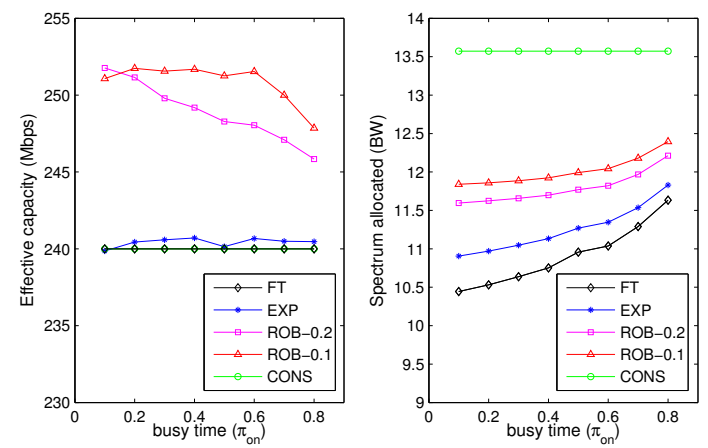

Fig. 5. Effective capacity and spectrum allocated as a function of $\pi_{\text {on }}$ when $\mathrm{p}_{\mathrm{on}}=0.01$ and $d=240 \mathrm{Mbps}$ (total unlicensed capacity $=248 \mathrm{Mbps}$ ). 


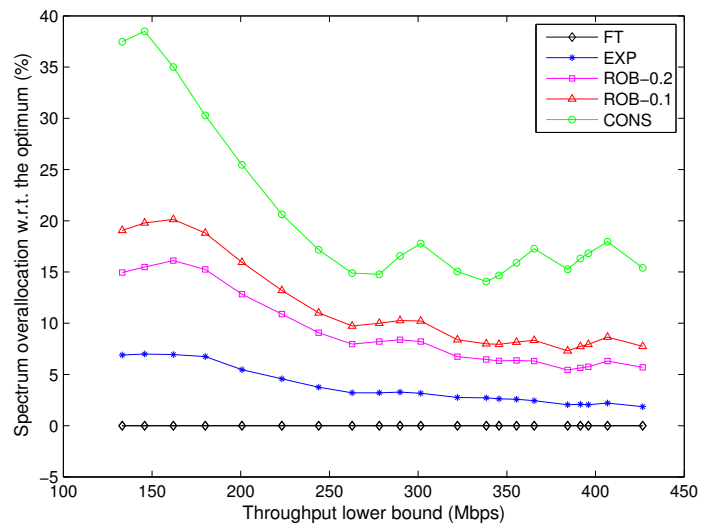

Fig. 6. Simulation results for $d=0.9 \cdot \sum_{u} c_{u}\left(\mathrm{p}_{\mathrm{on}}=0.01\right.$ and $\left.\pi_{\mathrm{on}}=0.1\right)$.

consider for each case a throughput lower bound equal to $90 \%$ of its value. In Fig. 6 we can see the spectrum overallocation compared to the FT optimum. The stochastic methods clearly outperform CONS, with higher advantage for lower minimum throughput requirements, which is a expected result, as in this case it corresponds to a situation with more licensed bands. As the proportion of unlicensed spectrum gets higher, the benefit from using available licensed bands is lower, but even in situations where it is greater than the licensed spectrum, is still worth using it for reaching greater spectral efficiency. When we look at the short scale performance, we can see again that the proposed robust approach clearly outperforms the expectation based solution. While ROB-0.1 achieves $87.3 \%$ of success and ROB- 0.2 reaches $83.6 \%$, EXP only gets a poor $59.7 \%$. Furthermore, the price for that performance is only between $5 \%$ to $10 \%$ more spectrum assigned than EXP, and between $10 \%$ to $20 \%$ more than the optimum lower bound defined by the FT solution.

\section{CONCLUSIONS AND FUTURE WORK}

The spectrum allocation was studied in a mixed scenario, with both type of frequency bands, licensed and non licensed. The problem was analyzed from the perspective of SUs, which might use licensed bands whenever available and unlicensed bands all the time. We developed a general stochastic formulation considering a periodically scheduled assignment. We proposed a novel robust approach to solve the problem and analyzed the advantage against an expectation based solution, comparing their performance by extensive simulations. The results show that the proposed solution presents much better performance with not much additional allocated spectrum.

For future work, we will extend our framework to a multiple-domain scenario, where we also have to consider inter-domain interference. The goal will be to analyze how the spectrum sharing between different domains affect the optimal spectrum allocation. This is the situation in a multihop wireless mesh network, where links between nodes might interfere with each other. In that case, an individual spectrum allocation by each node would lead to an inefficient solution, so some kind of coordination between nodes would be necessary. On the other hand, it would also be interesting to compare the periodically scheduled allocation proposed with an eventdriven solution. We could analyze which is the threshold in the PUs' dynamics when signaling overhead of the latter becomes tolerable to get a more efficient spectrum allocation.

\section{ACKNOWLEDGMENTS}

This work was partially supported by Centro Ceibal and ANII (grant POS_2011_1_3525).

\section{REFERENCES}

[1] E. Halepovic, C. Williamson, and M. Ghaderi, "Wireless data traffic: a decade of change," IEEE Network, vol. 23, no. 2, pp. 20-26, 2009.

[2] Cisco White Paper, "Cisco visual networking index: Global mobile data traffic forecast update, 2013-2018," February 2014.

[3] Plan Ceibal, "Equidad, Tecnología y Educación para el desarrollo humano," 2012.

[4] "Super Bowl XLVIII Stats Infographic - Extreme Networks." [Online]. Available: http://www.extremenetworks.com/super-bowl-stats/

[5] FCC Notice of Proposed Rulemaking 13-22, "Revision of Part 15 of the Commission's Rules to Permit Unlicensed National Information Infrastructure (U-NII) Devices in the 5 GHz Band," February 2013.

[6] J. Mitola, "Cognitive Radio - An Integrated Agent Architecture for Software Defined Radio," PhD thesis, Royal Institute of Technology (KTH), Kista, Sweden, May 2000.

[7] FCC Second Memorandum Opinion and Order 10-174, "Unlicensed Operation in the TV Broadcast Bands, Additional Spectrum for Unlicensed Devices Below $900 \mathrm{MHz}$ and in the $3 \mathrm{GHz}$ Band," September 2010.

[8] IEEE Std 802.22-2011 Standard for Wireless Regional Area Networks (RAN) - Part 22, "Cognitive Wireless RAN Medium Access Control (MAC) and Physical Layer (PHY) specifications: Policies and procedures for operation in the TV Bands," July 2011.

[9] "IEEE Standard for Information technology - Telecommunications and information exchange between systems - Local and metropolitan area networks - Specific requirements - Part 11: Wireless LAN Medium Access Control (MAC) and Physical Layer (PHY) Specifications Amendment 5: Television White Spaces (TVWS) Operation," IEEE Std 802.11af-2013, pp. 1-198, February 2014.

[10] E. Tragos, S. Zeadally, A. Fragkiadakis, and V. Siris, "Spectrum assignment in cognitive radio networks: A comprehensive survey," IEEE Communications Surveys \& Tutorials, vol. 15, no. 3, pp. 1108-1135, 2013.

[11] F. Novillo, M. Churchman, R. Ferrus, and R. Agusti, "A channel allocation algorithm for OSA-enabled IEEE 802.11 WLANs," in International Symposium on Wireless Communication Systems, September 2009.

[12] F. Liu, E. Erkip, M. C. Beluri, R. Yang, and E. Bala, "Dual-band femtocell traffic balancing over licensed and unlicensed bands." in International Conference on Communications, 2012.

[13] M. Timmers, S. Pollin, A. Dejonghe, L. V. der Perre, and F. Catthoor, "A distributed multichannel mac protocol for multihop cognitive radio networks," IEEE Trans. on Vehicular Technology, vol. 59, no. 1, pp. 446-459, 2010.

[14] A. Kashyap, S. Ganguly, and S. R. Das, "A measurement-based approach to modeling link capacity in 802.11-based wireless networks," in International Conference on Mobile Computing and Networking, 2007.

[15] J. Guerin, S. Glass, P. Hu, W. L. Tan, and M. Portmann, "Time-based and low-cost bandwidth estimation for IEEE 802.11 links," in International Wireless Communications and Mobile Computing Conference, August 2012.

[16] G. Calafiore and L. Ghaoui, "On distributionally robust chanceconstrained linear programs," Journal of Optimization Theory and Applications, vol. 130, no. 1, pp. 1-22, 2006.

[17] M. Lopez-Benitez and F. Casadevall, "Discrete-time spectrum occupancy model based on markov chain and duty cycle models," in New Frontiers in Dynamic Spectrum Access Networks (DySPAN), 2011 IEEE Symposium on, May 2011, pp. 90-99.

[18] M. Grant and S. Boyd, "CVX: Matlab software for disciplined convex programming, version 2.0 beta," http://cvxr.com/cvx, Sep. 2013.

[19] "The MOSEK optimization software." [Online]. Available: http://www.mosek.com/ 